\title{
Variable stars in nearby galaxies
}

\section{Frequency-period distribution of Cepheids in IC 1613 and other galaxies of the Local Group}

\author{
E. Antonello, D. Fugazza, and L. Mantegazza
}

Osservatorio Astronomico di Brera, Via E. Bianchi 46, 23807 Merate, Italy

Received 29 January 2002 / Accepted 29 March 2002

\begin{abstract}
The frequency-period distribution and other properties of Cepheids in IC 1613 are discussed and compared with those of stars in our Galaxy (Milky Way), LMC, SMC, M 31 and M 33. Taking into account the observational limitations and related incompleteness, it is concluded that the frequency-period distribution of Cepheids in IC 1613 is similar to that of SMC; we suspect that a much larger number of stars exist in IC 1613 with a period of less than $2 \mathrm{~d}$ that have not yet been detected. A discussion of the deficiency of fundamental mode Cepheids with periods in the range 8-10 d in the Milky Way, M 31 and M 33 is reported. The present data are not sufficient to verify if this is produced by a real bimodal frequency-period distribution or whether depends on the lack of pulsating stars in such a period range due to pulsational stability reasons. Some arguments are presented in favor of a bimodal distribution that is a function of the average metallicity. The Milky Way, M 31 and M 33 have the two maxima located at the same periods, about 5 and $13 \mathrm{~d}$, respectively. A comment on very long period Cepheids is also given.
\end{abstract}

Key words. stars: oscillations - stars: variables: Cepheids - galaxies: individual: IC 1613 - galaxies: Local Group - galaxies: stellar content

\section{Introduction}

Cepheids are variable stars that are used to measure distances of galaxies in the Local Group and nearby clusters, and are the primary calibrators for the secondary standard candles that are applied at much greater distances. However, they are important also for testing the theories on the internal constitution of stars and stellar evolution. In particular, the study of Cepheids in nearby galaxies is important to understand the effects of different metallicities and corresponding mass-luminosity relations on the pulsational characteristics. The purpose of our project was to obtain good light curves of Cepheids in IC 1613 and NGC 6822. In order to make best use of telescope time and reach fainter magnitudes, our strategy was to observe in white light, i.e. without filter ( $W h$ photometry). The results of the survey for variable stars in four fields of IC 1613 were reported in the previous papers of this series (see Mantegazza et al. 2001, Paper IV, and references therein), while the properties of population I and II Cepheids observed in a field were discussed by Antonello et al. (1999b, Paper II). Recently, the first results for NGC 6822 have also been presented (Antonello et al. 2002, Paper V). In

Send offprint requests to: E. Antonello,

e-mail: elio@merate.mi.astro.it

* Based on observations collected at ESO-La Silla. another paper (Antonello et al. 2000b), the light curves of the Cepheids with period near $10 \mathrm{~d}$ of IC 1613 were analyzed and compared with those of the corresponding objects in the Galaxy and in the Magellanic Clouds. In the present paper we summarize the characteristics of the Cepheids observed in IC 1613 and make a comparison with other galaxies containing a large number of such stars, taking into account the results of other recent surveys. We will update the discussion about observational evidence for a dip in the frequency-period distribution near 8-10 d, that is seen preferentially in metal rich galaxies. Presently there are three different interpretations of such dips.

1) In their extensive study on the frequency-period distribution of Cepheid variables, Becker et al. (1977; hereafter BIT) concluded that it is not compatible with a standard birthrate function, and can only be explained if an ad hoc two-component birthrate function is adopted. The primary component is a time-averaged "background" rate which reproduces the main peak of the distribution (e.g. that in the Milky Way). The second component is produced by recent star formation (e.g. in OB associations) and is characterized by some cutoff below a critical mass; this component reproduces the secondary peak and the cutoff explains the dip.

2) Chiosi (1989) noted that, since the blue band of core He-burning of models with relatively large mass 
( $\left.\gtrsim 9 M_{\odot}\right)$ tend to move back to the red giant region (a tendency enhanced by increasing metallicity), such masses would spend a large fraction of the blue band lifetime within the instability strip; therefore the excess of long $P$ Cepheids would be intrinsic to the stellar models and a two-component birthrate is not needed.

3) Buchler et al. (1997) suggested that the dip could be a real deficiency of stars caused by the instability of the nonlinear fundamental pulsation cycle in this period range, and such stars that cannot pulsate in the fundamental mode actually pulsate in the first overtone one.

We recall a wish expressed more than twenty years ago by $\operatorname{BIT~(p.~649)~in~relation~to~the~Cepheids~in~}$ the seven galaxies, Milky Way, LMC, SMC, M31, M33, IC 1613 and NGC 6822: "The Cepheids in M 33, NGC 6822 and IC 1613 have only begun to be studied, and much more work needs to be done. Further information on the frequency-period distribution of any of these seven galaxies would be very helpful".

\section{Cepheids in IC 1613}

\subsection{Generalities}

Details of the Wh CCD observations (performed with the ESO Dutch $0.9 \mathrm{~m}$ telescope) and reduction methods were reported in the previous papers of this series, along with the lists of variable stars and their coordinatae (Antonello et al. 1999a; Antonello et al. 2000a; Mantegazza et al. 2001). The finding charts of the variable stars are available upon request to the authors, or can be found in the Archive at the site http://www.merate.mi.astro.it/ fugazza/cepheids.html. The analysis of the $W h$ light curves was performed as in Paper II, and the Fourier parameters were used to identify the pulsation mode of shorter $P$ Cepheids. The mode classification of the objects for each field was reported in the previous papers; we have however revised it by using homogeneous criteria, and in particular we tried to classify the stars previously reported with uncertain modes. The result is that the fundamental $F$ mode pulsators include also the following stars: V0236A, V2991B, V3277B, V3782B, V0793D, V1307D, and the first overtone $1 O T$ pulsators include also the following stars: V0178A, V0524A, V1296A, V1289A, V0223B, V0987B. We recall that, owing to the uncertainties on the parameters, the mode identification for the shorter $P$ stars is only preliminary.

In the lower panel of Fig. 1 the $P L$ relation is shown for the whole set of stars, which includes $82 F$ mode, $341 O T$ mode, 3 suspected second overtone $2 O T$ mode pulsators, and 5 Cepheids with uncertain mode. In the upper panel we show the diagram of amplitude vs. $P$. The three $F$ mode Cepheids marked with a cross in a circle have the lowest amplitude and are the brightest stars among those with similar $P$; we suspect that they are binary systems or there is a blending effect. An analysis of all the Fourier parameters indicates that, for $P$ less

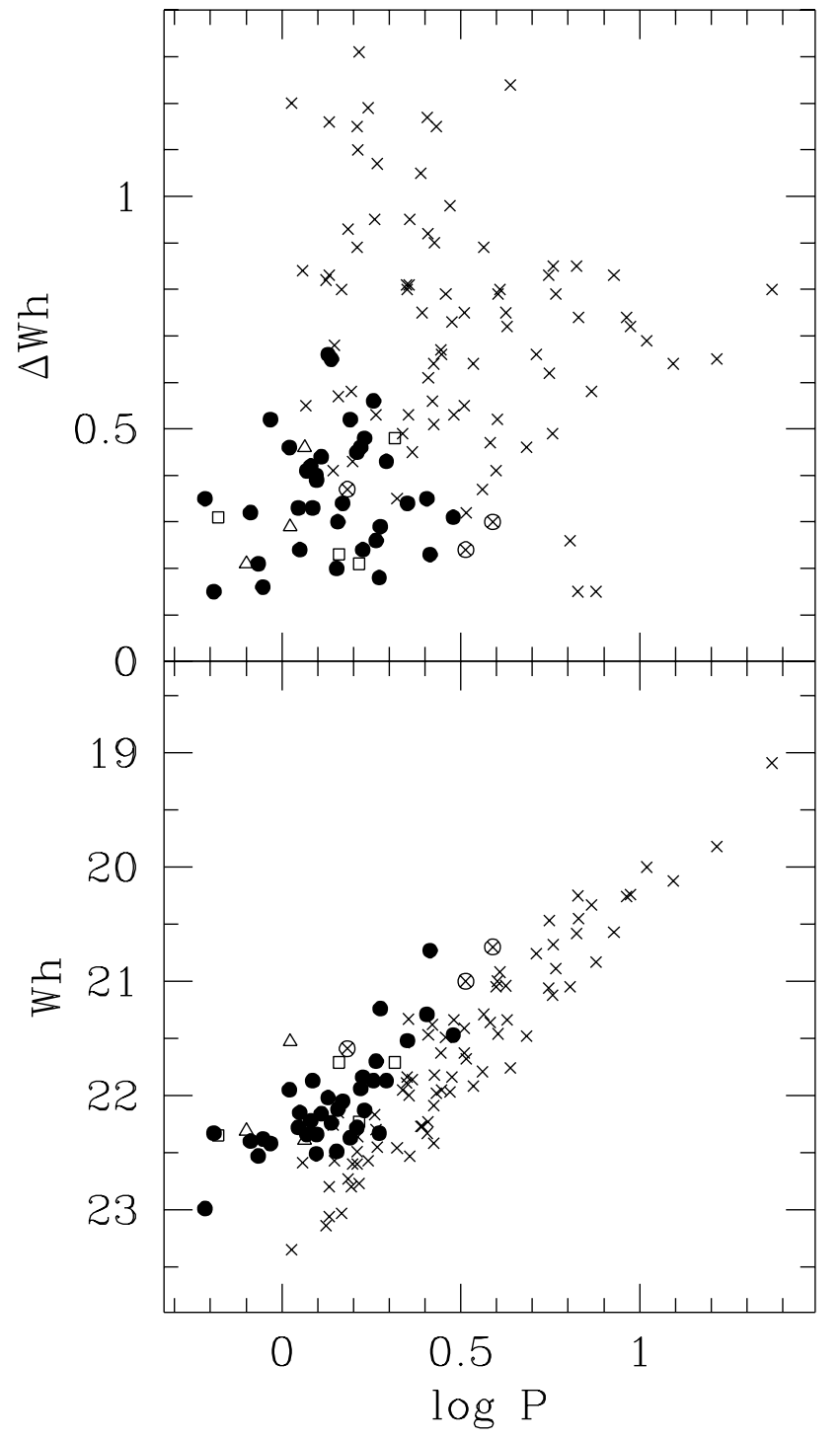

Fig. 1. Lower panel: PL relation for IC 1613 Cepheids. Crosses: fundamental mode; filled circles: first overtone mode; open squares: uncertain mode; open triangle: second overtone candidate; cross in circle: fundamental mode Cepheid with a probable companion. For a comparison with other surveys, we remark that $V \sim W h+0.3$ for stars with cepheid color. Upper panel: full amplitude against $P$.

than about $4 \mathrm{~d}$, the formal errors can be relatively large, and the resulting relation between the phase difference $\phi_{21}=\phi_{2}-2 \phi_{1}$ (of the second and first Fourier component) and $P$ is relatively scattered. Therefore, this does not allow us to compare reliably such parameters of short $P$ fundamental mode Cepheids in IC 1613 with those of similar stars in other galaxies, when looking for slight differences due to different metallicities. In other words, with the present data, useful comparisons should be limited to stars with $P \gtrsim 4$ d (e.g. Antonello et al. 2000b), instead of attempting a general comparison, as done in Paper II. 


\subsection{Comparison with OGLE survey}

Recently, the OGLE project made a survey of Cepheids in IC 1613, in the $V$ and $I$ bands (Udalski et al. 2001), using a $1.3 \mathrm{~m}$ telescope. Owing to the larger field of view (14!), they observed almost the whole galaxy and found about 135 population I Cepheids, with a limiting average magnitude $V \sim 22.6$. In the $V$ band the total exposure time was $900 \mathrm{~s}$, and the seeing conditions were much better than those of our project; in fact the Dutch $0.9 \mathrm{~m}$ telescope suffered from focusing problems. In our four selected fields we found about 128 Cepheids, with a limiting magnitude of $V \sim 23$. We verified that, in the same four fields, OGLE detected about 75 Cepheids; there are 64 Cepheids in common with us. The comparison shows that there are systematic differences of some arcsec in the declination and right ascension between OGLE and our lists. The $P$ values are reasonably in agreement. The largest discrepancies were found for three stars with $P$ close to $2 \mathrm{~d}$, in which case it is difficult to distinguish between the true $P$ and the alias produced by the one-day separation of observations. Indeed, in the power spectrum there is a significant peak at both $f=1 / P \mathrm{~d}^{-1}$ and $f=1-1 / P \mathrm{~d}^{-1}$. We merged our and OGLE dataset after correcting for the different mean magnitude and different amplitude, and we got for $V 0122 B=O G L E 12044$ $2.032 \mathrm{~d}$, for $V 0987 B=O G L E 052092.094 \mathrm{~d}$, and for $V 0231 C=O G L E 010921.802 \mathrm{~d}$.

We also analysed several merged data sets, containing about $90-100 \mathrm{Wh}$ and $V$-corrected data points each, but we were not able to improve the accuracy of the light curve Fourier parameters of shorter $P$ stars. In particular we cannot confirm the possible indications for the resonance effects in $1 O T$ pulsators reported in Paper II. The conclusion is that the accurate study of the light curves of Cepheids fainter than $V \sim 22$ requires a larger telescope than that adopted by us or by the OGLE project.

Finally we mention the deep HST imaging of a field in the halo of IC 1613 performed by Dolphin et al. (2001); they found 11 short $P$ Cepheids and 13 RR Lyrae stars.

\section{Period distribution}

The $P$ distribution is shown in Figs. 2, 3 and 4 and is compared with those of other galaxies in the Local Group. In Fig. 2 the histogram of all the classical Cepheids is reported, while in Fig. 3 the stars are separated according to the pulsation mode; finally, Fig. 4 shows the homogeneous distribution when the $P$ of $1 O T$ pulsators is multiplied by an average period ratio $P_{0} / P_{1} \sim 1 / 0.7$. The adopted bin size is $\Delta \log P=0.05$; for IC 1613 in Figs. 3 and 4 it is $\Delta \log P=0.1$. Since we are interested in pointing out the new results with respect to previous studies, the histograms are focused on intermediate and shorter $P$ Cepheids $(P \lesssim 50 \mathrm{~d}$ ); a comment on longer $P$ stars is however reported as a conclusive note in the Discussion.

$I C$ 1613. The histogram shown in Fig. 2 includes all the known 209 Cepheids, while the sample in Figs. 3 and 4 is based on our survey and it includes the stars found by Dolphin et al. (2001). The samples should be considered almost complete for $P$ longer than about $2-2.5 \mathrm{~d}(\log P \sim$ 0.4). Probably there are no $1 O T$ Cepheids with $P \gtrsim 3 \mathrm{~d}$.

Milky Way. For the histogram in Fig. 2 we considered 504 stars in the Galactic Cepheid Database (Fernie et al. 1995). For Fig. 3 we have used the results on the 348 stars with good light curves analyzed by the BreraMerate group (e.g. Poretti 1994; Antonello \& Morelli 1996, and references therein), which include 36 identified $1 O T$ pulsators. We recall that it is possible to discriminate between $1 O T$ mode and $F$ mode stars using light curve Fourier parameters only when the $P$ is shorter than about $5.5 \mathrm{~d}$, since above this value the light curves tend to be similar. Therefore no identified $1 O T$ mode stars exist with longer $P$. On the other hand, the identified $1 O T$ Cepheids whose distribution is shown in Fig. 3 should constitute a fairly complete sample of these stars with $P \lesssim 5.5 \mathrm{~d}$ in the vicinity of the Sun.

$L M C$ and $S M C$. The data for stars in LMC and SMC were taken from OGLE data archive (Udalski et al. 1999a; Udalski et al. 1999b; Udalski et al. 1999c; Soszynski et al. 2000); they include also the double mode Cepheids (DMC). For SMC we have $1343 F$ stars (24 of them are DMCs pulsating in $F$ and $1 O T$ mode), and $8901 O T$ stars (71 DMCs pulsating in $1 O T$ and $2 O T$ mode). For the LMC we have $791 F$ stars (20 DMCs pulsating in $F$ and $1 O T$ mode), and $5691 O T$ stars (61 DMCs pulsating in $1 O T$ and $2 O T$ mode). Owing to OGLE observational constraints, the sample does not contain stars with $P$ longer than about $40 \mathrm{~d}$; however this is not an issue for the problem we will discuss here. On the other hand, the sample is fairly complete as regards the shorter $P$ stars, and is well representative of the Cepheid population in the surveyed fields of the LMC and SMC. The distributions of LMC Cepheids show a long tail at shorter $P$, given by $1 O T$ pulsators.

M 31. After an analysis of DIRECT M 31 data archive (Mochejska et al. 1999 and references therein), we preferred to adopt the previous results on 392 stars reported in the GCVS (Samus 1995). The reason is that DIRECT sample appears incomplete for $P$ shorter than about $6 \mathrm{~d}$, where the old data distribution shows a prominent maximum; the difference is not due to possible different observed regions of the galaxy. As discussed by BIT, the sample produced by the photographic data should be considered representative for $\log P \gtrsim 0.7$. We note in passing that there is not yet information available on the existence of $1 O T$ pulsators in this galaxy.

M 33. For M 33 we adopted the results of the DIRECT survey published by Macri et al. (2001), Mochejska et al. (2001a) and Mochejska et al. (2001b); the Cepheid lists were checked for the presence of the same star in different lists, on the basis of stellar coordinatae. The sample contains 654 stars. Given the adopted observational techniques, the Cepheids with $P>14 \mathrm{~d}$ are partially underestimated. On the whole, keeping in mind this possible selection effect, we think the sample is representative of 
the Cepheid population for $\log P \gtrsim 0.5$. Unfortunately the authors do not report the mode classification, even if uncertain; the $P L$ relation for M 33 shows the very probable presence of $1 O T$ mode pulsators with $P$ up to $7-8 \mathrm{~d}$. We have adopted the separation line reported in their Fig. 11 for discriminating between $F$ and candidate $1 O T$ mode stars. Of course, the results of the analysis should be considered only as preliminary.

\section{Discussion and conclusion}

We are trying to compare the frequency-period distribution in different galaxies even if the samples have different degrees of completeness. The possible selection effects that hinder attempts to achieve complete samples were discussed by BIT, and we can revise them, taking into account the progresses made in the last twenty years. Variables with large amplitude variations are more likely to be discovered than those with small amplitude variations. Since the amplitude is related to the $P$, the discovery of stars in the $P$ ranges where the amplitude is larger is favored. The photographic technique made it difficult to find Cepheids with amplitudes less than about $0.5 \mathrm{mag}$; for the CCD technique this limit is much lower (for example, this has allowed the detection of $1 O T$ pulsators in galaxies beyond the Magellanic Clouds). The main selection effect is therefore the limiting magnitude of each survey, coupled with the detectable amplitude (which is related to the error of the measurements). This favors the selection of Cepheids with longer $P$ since they are brighter, while the detectable Cepheids with shorter $P$ and for a given pulsation mode tend to be those with larger amplitudes. In Sect. 3 we have reported for each galaxy the estimated shorter $P$ limit, and a reliable comparison between galaxies can be done above such a value. Finally, we mention the ever-present problem of discovering variables with $P$ close to $1 \mathrm{~d}$; this difficulty is related to the constraint of observing at one-day intervals.

Many of the differences among the various galaxies in Figs. 2, 3 and 4 can be interpreted in terms of different average metallicity. According to the data collected by van den Bergh (2000), the metallicity indicator values (for young population I stars) can be summarized as in Table 1. As is well known (see e.g. BIT), the location of the maximum of the frequency-period distribution increases with increasing metallicity.

As regards IC 1613, taking into account the incompleteness of the sample, the distributions are in part similar to those of SMC. The maximum of the distribution in Fig. 2 would be probably located at $P \lesssim 1.6 \mathrm{~d}$ $(\log P \lesssim 0.2)$, and we should expect a larger number of stars with $P \lesssim 2$ d yet to be discovered. The peak at $\log P=0.4$ is intriguing, but we cannot give it much weight. It is interesting to compare the LMC and SMC in Figs. 2 and 4; after roughly correcting for the different pulsation modes, the shape difference of the distributions (Fig. 2) decreases significantly (Fig. 4). On the other hand, the similarity of the shape of the distributions of $1 O T$ and

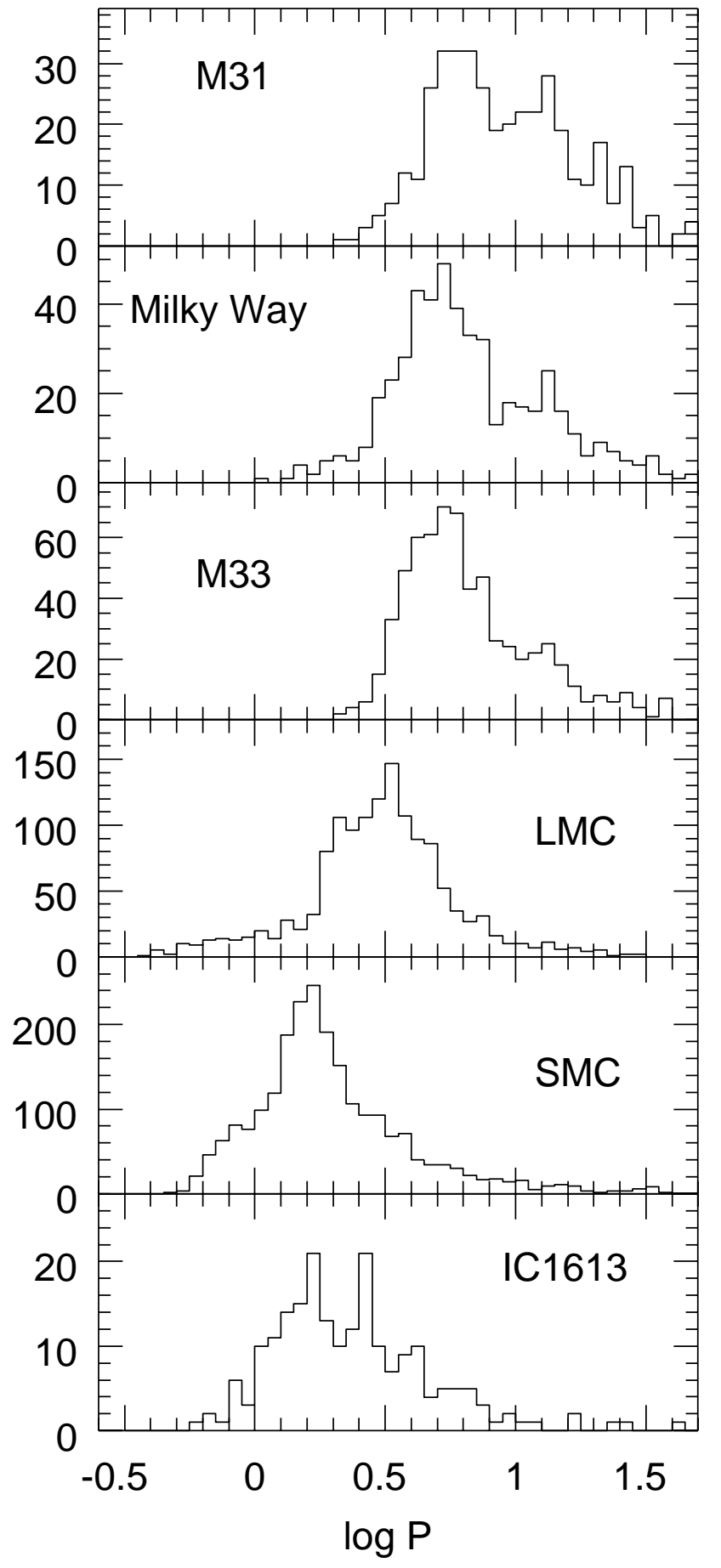

Fig. 2. Period distribution of Cepheids in galaxies of the Local Group.

$F$ pulsators in LMC (Fig. 3) appears rather unique among galaxies.

In spite of the different metallicities, the location of primary and secondary maxima of M31, Milky Way and M 33 are practically the same. We can see in Fig. 2 that they are located at about $5 \mathrm{~d}(\log P \sim 0.7)$ and $13 \mathrm{~d}$ $(\log P \sim 1.1$; more exactly, between 12.6 and $14.1 \mathrm{~d})$, respectively. The recent observations of M 33 made by the DIRECT project give us the opportunity to update the 


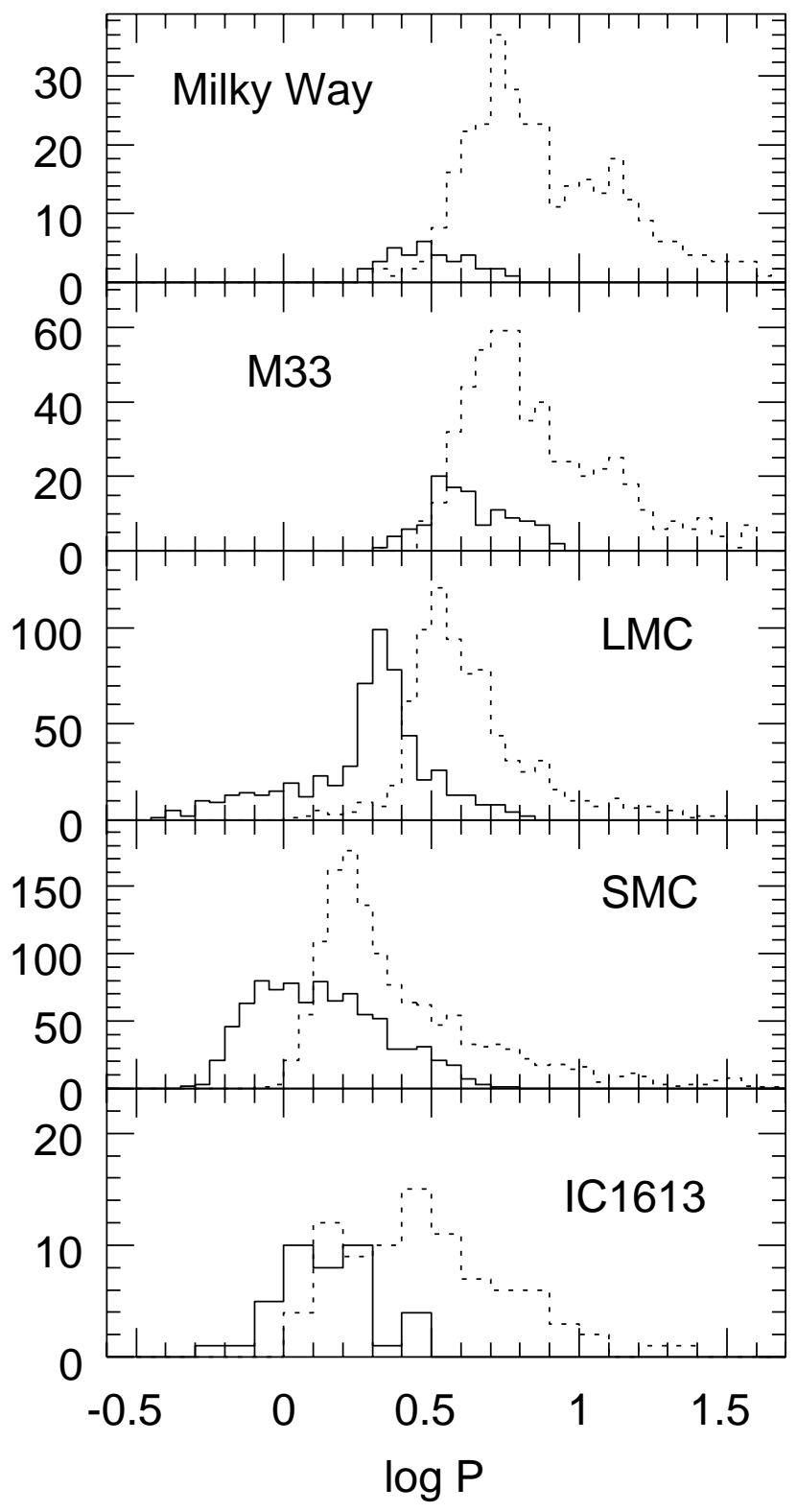

Fig. 3. Period distribution of Cepheids pulsating in $F$ (dotted line) and $1 O T$ (continuous line) mode.

study on the possible origin of the dip located at about 8-10 d $(\log P \sim 0.9-1.0)$. According to the interpretation of Buchler et al. (1997), in the Milky Way a deficiency of $F$ mode pulsators in this $P$ range is expected owing to the instability of the pulsation cycle; since such stars will actually pulsate in the $1 O T$ mode, the deficiency must be compensated by an excess of $1 O T$ pulsators in the $P$ range of about 5.6-7 d. As noted in the previous Sect. 3 (Milky Way), it is not possible to discriminate the pulsation mode for $P \gtrsim 5.5 \mathrm{~d}$ using light curve parameters alone, therefore for the present we cannot say how many $1 O T$ pulsators are actually located between $\log P \sim 0.75$ and $\sim 0.84$ in the Milky Way. In M 33, according to the $P L$ diagram reported by Mochejska et al. (2001b), there are some probable $1 O T$ stars located in such $P$ range (Fig. 3 ), but it is difficult to affirm that there exists an excess of $1 O T$ stars;

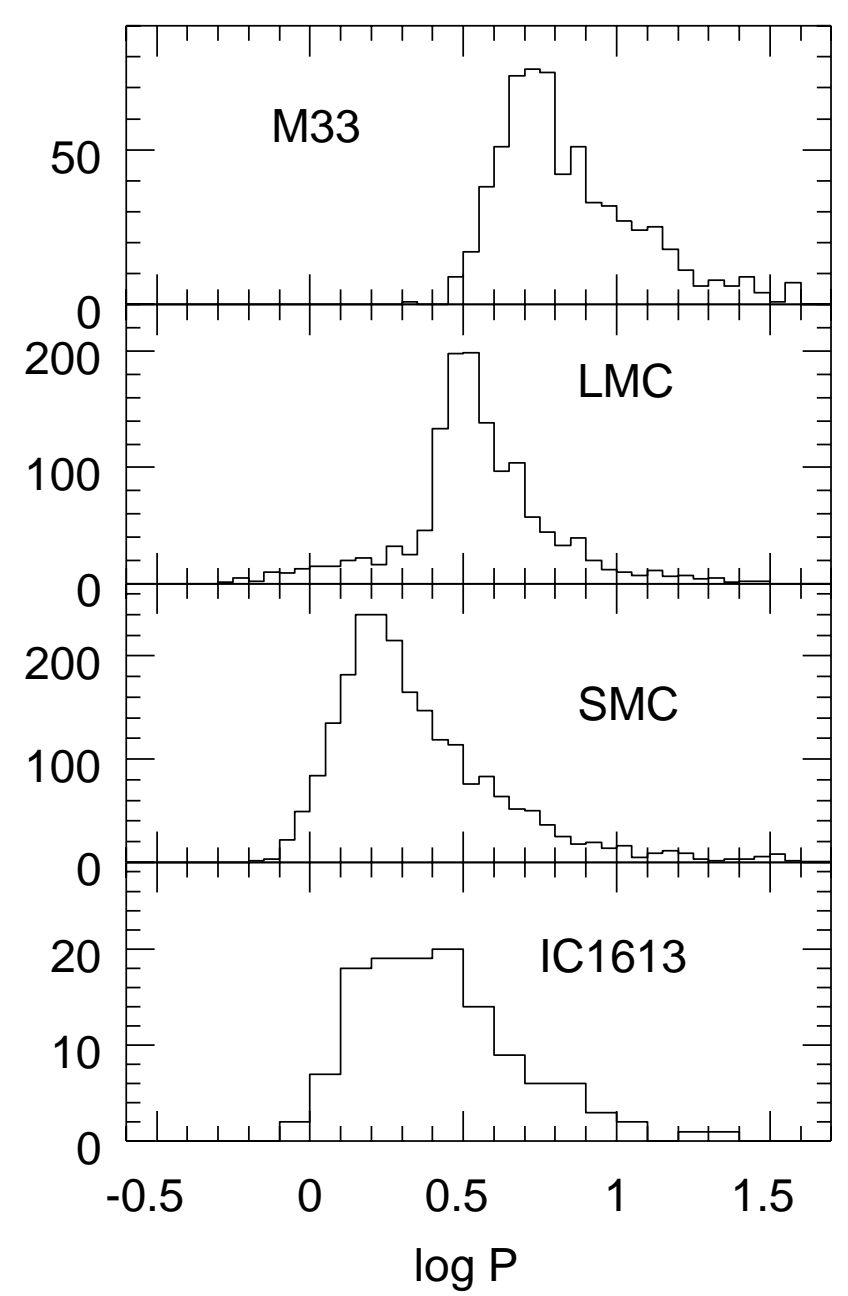

Fig. 4. Homogeneous period distribution for $F$ and $1 O T$ mode Cepheids; the $P$ of $1 O T$ pulsators was multiplied by a factor $1 / 0.7$, which is an average value of the ratio $P_{0} / P_{1}$ (see text).

on the other hand also the dip, which is located near $10 \mathrm{~d}$, is not very pronounced. The exercise shown in Fig. 4 indicates that indeed the dip tends to be filled by the $1 O T$ pulsators, if we change their $P$ to that of the $F$ mode one, but in any case there is still a bimodal distribution, i.e. there is an excess of stars with $\log P \sim 1.1$ which must be explained. Finally, it is instructive to look at M31 even if there is no information on the $1 O T$ pulsators. In this case, even assuming a certain number of $1 O T$ pulsators with $P$ within $5.6-7 \mathrm{~d}$ which more or less fill the dip, they will never cancel the evident bimodal distribution. It is important to remark that we do not exclude the effect proposed by Buchler et al. (1997). We just say that it does not look sufficient to explain the bimodal distribution.

It is difficult to verify which of the two interpretations, BIT or Chiosi's (1989) one, would be more acceptable. Roughly speaking, M33 data confirm the expectation of both that the secondary component depends on the metallicity of the galaxy. If we make the reasonable assumption that the samples are fairly complete for a sufficiently long $P$ (e.g. above 6-7 d), and we consider normalized 
Table 1. Values of metallicity indicators of galaxies of the Local Group (young stars; van den Bergh 2000).

\begin{tabular}{lll}
\hline \hline galaxy & {$[\mathrm{Fe} / \mathrm{H}]$} & $12+\log (\mathrm{O} / \mathrm{H})$ \\
\hline M 31 & & 9.0 \\
Milky Way & +0.06 & 8.7 \\
M 33 & & 8.4 \\
LMC & -0.30 & 8.37 \\
SMC & -0.73 & 8.02 \\
IC 1613 & -1.3 & 7.86 \\
\hline
\end{tabular}

distributions of M33, Milky Way and M31, the data indicate that this component increases montonically with the metallicity. Then the question is which of the two hypotheses predicts this relationship. The answer requires a more detailed study.

The clarification of some of these problems requires more accurate photometric data of Cepheids in the Local Group galaxies. Moreover, the longer $P 1 O T$ Cepheids in the Milky Way have yet to be identified; a useful technique for such an identification could be the analysis of the radial velocity curves. The preliminary results obtained by Moskalik \& Ogloza (2000) suggest indeed that the Fourier parameters of radial velocity curves and also the phase lag between light and radial velocity curves could be mode discriminators for $P \gtrsim 5 \mathrm{~d}$. This is a necessary study before beginning a reliable discussion of the Cepheids in the Milky Way. Unfortunately, we think there is a further dilemma: assuming the excess of $1 O T$ pulsators does exist, should it be the expected signature of the pulsational stability or would it be just the bimodal distribution of the $1 O T$ pulsators?

As a final remark we note that, with respect to BIT, there are few new observational results on very long $(P>100 \mathrm{~d})$ Cepheids. The HST Key Project on $H_{0}$ determination was a good opportunity for a statistics on a large sample of galaxies, but unfortunately the observations were limited to periods shorter than 100 d. According to BIT, Cepheids with $\log P>2.0$ are probably massive stars burning carbon in their cores, and those authors infer that a whole galaxy such as M31 would have to be searched to find just one Cepheid in the core-carbon-burning phase. However, these stars are present in dwarf irregular galaxies such as LMC, SMC, IC 1613 and, as recently observed, also in NGC 6822 (Antonello et al. 2002). BIT interpreted the discrepancy as further support for their suggestion of a two-component birthrate function, the second component giving 5-50 times as many massive stars as indicated by the one-component model. However, the nonlinear pulsation characteristics could also play a role in this case. Aikawa \& Antonello (2000) found that the nonlinear pulsation cycle for $P>100 \mathrm{~d}$ is stable only for low metallicity values, therefore the very long $P$ Cepheids should be preferably detected in metal-poor galaxies such as dwarf irregulars. This would be a simple explanation of what is actually observed.

\section{References}

Aikawa, T., \& Antonello, E. 2000, A\&A, 363, 601

Antonello, E., Mantegazza, L., Fugazza, D., Bossi, M., \& Covino, S. 1999a, A\&A, 349, 55 (Paper I)

Antonello, E., Mantegazza, L., Fugazza, D., \& Bossi, M. 1999b, A\&A, 350, 797 (Paper II)

Antonello, E., Fugazza, D., Mantegazza, L., Bossi, M., \& Covino, S. 2000a, A\&A, 363, 29 (Paper III)

Antonello, E., Fugazza, D., \& Mantegazza, L. 2000b, A\&A, 356, L37

Antonello, E., Fugazza, D., Mantegazza, L., Stefanon, M., \& Covino, S. 2002, A\&A, 386, 860 (Paper V)

Antonello, E., \& Morelli, P. L. 1996, A\&A, 314, 541

Becker, S. A., Iben, I., \& Tuggle, R. S. 1977, ApJ, 218, 633 (BIT)

Buchler, J. R., Goupil, M. J., \& Piciullo, R. 1997, ApJ, 491, L102

Chiosi, C. 1989, in The Use of Pulsating Stars in Fundamental Problems in Astronomy, ed E. G. Schmidt, IAU Colloq., 111 (Cambridge Univ. Press), 39

Dolphin, A. E., Saha, A., Skillman, E. D., et al. 2001, ApJ, 550,554

Fernie, J. D., Beattie, B., Evans, N. R., \& Seager, S. 1995, IBVS, 4148

Macri, L. M., Stanek, K. Z., Sasselov, D. D., Krockenberger, M., \& Kaluzny, J. 2001, AJ, 121, 870

Mantegazza, L., Antonello, E., Fugazza, D., Bossi, M., \& Covino, S. 2001, A\&A, 367, 759 (Paper IV)

Mochejska, B. J., Kaluzny, J., Stanek, K. Z., Sasselov, D. D., \& Szentgyorgyi, A. H. 2001a, AJ, 121, 2032

Mochejska, B. J., Kaluzny, J., Stanek, K. Z., Sasselov, D. D., \& Szentgyorgyi, A. H. 2001b, preprint [astro-ph/0107279] to appear in AJ

Mochejska, B. J., Kaluzny, J., Stanek, K. Z., Krockenberger, M., \& Sasselov, D. D. 1999, AJ, 118, 2211

Moskalik, P., \& Ogloza, W. 2000, in The Impact of Large-Scale Surveys on Pulsating Star Research, ed. L. Szabados, D. W. Kurtz (ASP) 203, 237

Poretti, E. 1994, A\&A, 285, 524

Samus, N. N. 1995, General Catalogue of Variable Stars, Volume V (Moscow: Kosmosinform)

Soszynski, I., Udalski, A., Szymanski, M., et al. 2000, AcA, 50, 451

Udalski, A., Soszynski, I., Szymanski, M., et al. 1999a, AcA, 49,45

Udalski, A., Soszynski, I., Szymanski, M., et al. 1999b, AcA, 49, 223

Udalski, A., Soszynski, I., Szymanski, M., et al. 1999c, AcA, 49,437

Udalski, A., Wyrzykowski, L., Pietrzynski, G., et al. 2001, preprint [astro-ph/0109446]

van den Bergh, S. 2000, The Galaxies of the Local Group, Cambridge Astrophys. Ser. 35 (Cambridge Univ. Press) 